\title{
Erratum to: Probiotic Therapy for Treating Behavioral and Gastro- intestinal Symptoms in Autism Spectrum Disorder: A Systematic Review of Clinical Trials
}

Jun LIU ${ }^{1 \dagger}$, Guo-bin WAN ${ }^{2 \dagger}$, Ming-shi HUANG ${ }^{2}$, George Agyapong ${ }^{1}$, Tian-le ZOU ${ }^{3}$, Xue-ying ZHANG ${ }^{4}$, Yen-Wenn LIU ${ }^{5}$, Yi-qing SONG 6 , Ying-Chieh Tsai ${ }^{5}$, Xue-jun $\mathrm{KONG}^{\text {7\# }}$

${ }^{1}$ Harvard Medical School, Boston 02115, USA

${ }^{2}$ Shenzhen Maternity \& Child Healthcare Hospital, Shenzhen 518048, China

${ }^{3}$ New York University, Buffalo 14214, USA

${ }^{4}$ Icahn School of Medicine at Mount Sinai, New York City 10029, USA

${ }^{5}$ National Yang Ming University, Taipei 112, China

${ }^{6}$ Indiana University, Indianapolis 46202, USA

${ }^{7}$ Massachusetts General Hospital, Charlestown 02129, USA

(C) Huazhong University of Science and Technology 2019

Erratum to: Current Medical Science

DOI https://doi.org/10.1007/s11596-019-2016-4

The original version of this article unfortunately contained two mistakes. The name and the work address of one author are wrong. The corrected name and work address are given below.

Guo-bin $\mathrm{WAN}^{2 \dagger}$

${ }^{2}$ Shenzhen Maternity \& Child Healthcare Hospital, Shenzhen 518048, China 